\title{
Zeit (zurück)geben - Momo und die grauen Herren
}

Pierre Bühler

Notre temps le temps n'est jamais devant Le seul temps qui fut c'est le temps perdu

Notre temps le temps n'est jamais devant Le seul temps qui fut c'est le temps reçu

Notre temps le temps n'est jamais devant Le seul temps qui fut c'est le temps vécu

Véronique Pestel, Le temps

Wenn zum 60. Geburtstag des Rektors über eine Kindergeschichte geschrieben wird, geschieht das nicht, um anzudeuten, dass man in diesem Alter langsam wieder kindisch wird, sondern vielmehr um darauf hinzuweisen, dass für einen Sechzigjährigen das bekannte Jesuswort weiterhin gilt: "Wenn ihr nicht umkehrt und werdet wie die Kinder, so werdet ihr nicht ins Reich der Himmel kommen". (Matthäus 18,3)

$\mathrm{Ob}$ die hier kommentierte Kindergeschichte zugleich zu einem Gleichnis für das Universitätsleben werden kann, dem sich der Rektor ganz besonders widmet, das sei der Fantasie der universitären Leserinnen und Leser überlassen. Es genügt, diese ab und zu bei der Wahrnehmung von Bezügen durch knappe Anspielungen zu stützen.

\section{Das Geheimnis der Zeit}

»Es gibt ein grosses und doch ganz alltägliches Geheimnis. Alle Menschen haben daran teil, jeder kennt es, aber die wenigsten denken je darüber nach. Die meisten Leute nehmen es einfach so hin und wundern sich kein bisschen darüber. Dieses Geheimnis ist die Zeit«. So beginnt das sechste Kapitel in Michael Endes Buch Momo ${ }^{1}$.

1 M.Ende, Momo oder Die seltsame Geschichte von den Zeit-Dieben und von dem Kind, das den Menschen die gestohlene Zeit zurückbrachte. Ein Märchen-Roman. 
Was macht die Zeit zum Geheimnis? Zunächst ist es die Tatsache, dass ihre Messung durch Kalender und Uhren wenig besagt, weil eine einzige Stunde wie eine Ewigkeit vorkommen oder wie ein Augenblick vergehen kann, je nachdem, was in dieser Stunde erlebt wird. »Denn Zeit ist Leben. Und das Leben wohnt im Herzen«. (61) Deshalb gilt auch viel grundsätzlicher, dass man nicht über sie verfügen kann, dass man sie nie im Griff hat, trotz aller Agenden, in denen sie geplant, organisiert, zerstückelt wird. Diese Unverfügbarkeit kommt in seltsamen Paradoxien zum Ausdruck, wie etwa beim Zeitsparen: »... je mehr die Menschen daran sparten, desto weniger hatten sie«. (78)

Deshalb stellt sich auch die geheimnisvolle Frage: Was geschieht, wenn die Zeit den Menschen verloren geht, wenn sie ihnen genommen, gestohlen wird, so dass sie dauernd nach Zeit haschen, schnappen müssen, um Zeit ringen müssen? Denn das passiert einem ja immer: Zeit ist immer schon verlorene Zeit. Ist diese verlorene Zeit unwiederbringlich verloren, oder kann sie den Menschen zurückgegeben werden? Gibt es auch da ein Geheimnis, so dass man sich nicht einfach die fehlende Zeit nehmen kann, dass sie einem nur gegeben, zurückgegeben werden kann? Davon handelt Michael Endes Kindergeschichte, und wer sie liest, kann es, während er sie liest, selbst erfahren.

\section{Das Geheimnis der grauen Herren}

Der Roman spielt in einer grossen Stadt, in der viele Menschen leben. In letzter Zeit kann beobachtet werden, dass diese Leute immer geschäftiger werden. Am unmittelbarsten bekommen es die Kinder zu spüren, denn die Erwachsenen haben für sie immer weniger Zeit übrig, so dass sie vernachlässigt, sich selbst überlassen und zugleich mit kostbaren Spielzeugen vertröstet werden. Die Menschen stehen unter einem Zwang, immer schneller zu gehen, immer schneller zu fahren, immer schneller zu arbeiten, immer schneller zu leben. Dabei aber scheint ihnen die Zeit immer mehr zu entgehen, so dass sie noch schneller gehen, fahren, arbeiten, leben müssen, wie in einer unendlichen Spirale gefangen.

Was ist mit den Menschen los? Die erzählerische Hypothese des Romans ist folgende: Insgeheim sind graue Herren am Werk. Angezogen sind sie mit grauen Anzügen und grauen Hüten (und

Mit Bildern des Autors, Stuttgart/Wien, (1973) 2005. Das Zitat steht auf S. 61. Für die weiteren Zitate steht die Seitenangabe im Text in Klammern. 
unter den Hüten haben sie glänzende Glatzen!). Sie rauchen graue Zigarren und tragen graue Aktentaschen. Wenn sie fahren, fahren sie in grauen Wagen. Überall wo sie auftauchen, stellt sich augenblicklich Kälte ein. Man sieht sie nur selten, weil sie ihr Werk am liebsten unerkannt, im Verborgenen vollziehen. Manchmal sieht man aber einen vorbeihuschen. Vielleicht auch in den Gängen der Universität? Da diese heute rauchfrei ist, sind die Zigarren weniger üblich geworden ... Aber sonst?

Es ist nicht ganz klar, woher diese grauen Herren kommen. Anscheinend haben die Menschen sie entstehen lassen, weil sie allzu unbesonnen, gedankenlos mit ihrer menschlich allzumenschlichen Zeit umgingen. Und nun haben diese Herren unter den Menschen leider auch Helfershelfer. Aber eigentlich stammen sie aus dem Nichts. Um leben zu können, um sich nicht wieder ins Nichts aufzulösen, woher sie kommen, müssen sie den Menschen dauernd ihre Zeit stehlen, denn nur von dieser menschlichen Zeit können sie leben. Und weil sie Zeitvorrat brauchen, arbeiten sie alle für eine "Zeit-Spar-Kasse«. Dort können sie sich auch stets wieder die nötige Zeit holen.

Man wird später im Roman vernehmen, dass es das Ziel der grauen Herren ist, die ganze Zeit aller Menschen in Besitz zu nehmen. Was dann aus den Menschen wird, ist nicht ihre Sorge. »Menschen ... sind längst überflüssig. Sie selbst haben die Welt so weit gebracht, dass für ihresgleichen kein Platz mehr ist. Wir werden die Welt beherrschen!« (252) Diese Herrschaft greift immer mehr um sich, und immer mehr Menschen fallen ihr zum Opfer. Wer könnte dagegen ankämpfen?

\section{Das Geheimnis Momos}

Da gibt es ein seltsames, kleines Mädchen namens Momo. Sie lebt am Rande der grossen Stadt, in der Ruine eines Amphitheaters, wo sie sich eine einfache Nische eingerichtet hat. Klein und ziemlich mager, mit einem wilden, pechschwarzen Lockenkopf und ebenso pechschwarzen Augen, ist sie ärmlich angezogen und geht meistens barfuss. Sie lebt mit dem, "was sie irgendwo fand oder geschenkt bekam« (8). Als sie Leute fragen, ob sie denn kein Zuhause habe, sagt sie, sie sei im Amphitheater zu Hause. Auf die Frage nach ihren Eltern antwortet sie, indem sie mit einer unbestimmten Handbewegung in die Ferne weist. Wann sie denn geboren sei, fragen die Leute: "Soweit ich mich erinnern kann, war ich immer schon da«. (9) Auf 
die Frage nach ihrem Alter antwortet sie: "Hundert«, und als die Leute nochmals fragen: "Also, ernsthaft, wie alt bist du? «, antwortet sie: "Hundertzwei«. (10)

Momo besitzt fast nichts, weil sie auch fast nichts braucht. Sie hat aber gute Freunde: Da ist der etwas schweigsame und besinnliche Beppo Strassenkehrer und der lebensfrohe und geschwätzige Gigi Fremdenführer, und da sind viele Kinder, die sie besuchen, um mit ihr zu spielen. Und Erwachsene auch, um mit Momo zu sprechen, denn sie hat eine Begabung, die allen, die ihr begegnen, gleich auffällt: "Was die kleine Momo konnte wie kein anderer, das war: zuhören. ... Wirklich zuhören können nur ganz wenige Menschen. Und so wie Momo sich aufs Zuhören verstand, war es ganz und gar einmalig«. (14) Bei Momos Zuhören werden die Menschen gescheit, entschlossen oder froh.

Doch nun kommen die Leute nicht mehr zu Momo, weil sie keine Zeit mehr haben. Spielen, Sprechen, Zuhören, wie es im Amphitheater gepflegt wird, all das ist Zeitverschwendung. "Früher sind die Leute gern zu Momo gekommen, damit sie ihnen zuhört. Sie haben sich dabei selbst gefunden, wenn ihr versteht, was ich meine. Aber jetzt fragen sie danach nicht mehr viel«. (86) Momo ist besorgt. Sie geht der Sache nach, versucht, mit den Freunden ins Gespräch zu kommen, sie von der tötenden Geschäftigkeit abzubringen. Und so fällt sie denn auch den grauen Herren auf, denn was im Amphitheater geschieht, kommt ihnen schon lange in die Quere. Eines Tages kommt einer der grauen Herren auf Besuch zu ihr, um sie zu fangen. Er versucht ihr zu zeigen, dass immer mehr und immer perfektioniertere Spielzeuge (von Bibigirl bis Bubiboy!) ein Kind von der Langeweile befreien: "Man muss nur immer mehr und mehr haben, dann langweilt man sich niemals". (100) Doch das Problem ist ja gerade, dass Momo sich gar nicht langweilt: Sie hat ja ihre Freunde, mit denen sie spielen und sprechen kann. Der graue Herr setzt nun etwas tiefer an: „Das Einzige, ... worauf es im Leben ankommt, ist, dass man es zu etwas bringt, dass man was wird, dass man was hat. Wer es weiterbringt, wer mehr wird und mehr hat als die anderen, dem fällt alles Übrige ganz von selbst zu ...«. (103)

Momos Geheimnis ist das Zuhören. Beim Agenten BLW/553/c (das ist die Identität des grauen Herrn!) fällt es ihr zunächst schwer. Es wird ihr in seiner Nähe immer kälter. Und sie hat beim Zuhören das Gefühl, ins Dunkle und Leere zu stürzen, »als sei da gar niemand «. (103) Doch mit Mut entschliesst sie sich, sich in die Dunkelheit und Leere des grauen Herrn zu stürzen, und dadurch kommt es zu einer Offenbarung: Der graue Herr gesteht ihr röchelnd, was die Absicht 
der grauen Herren ist, wie sie den Menschen die Zeit aussaugen, um selbst davon zu leben, und dass niemand wissen darf, dass es sie gibt und was sie tun. Plötzlich realisiert der Agent, dass sie ihn durch ihr Zuhören ausgehorcht hat, ihm das Geheimnis der grauen Herren entlockt hat, und er stürzt davon, seine Sachen eilend in sein graues Auto packend.

Durch Momos Zuhören offenbart sich das dunkle Geschäft ihrer Feinde.

\section{Das Geheimnis Kassiopeias}

Um die grauen Herren zu bekämpfen, muss ihr dunkles Geheimnis öffentlich bekannt gemacht werden. Momo und ihre Freunde entscheiden sich, durch eine Demonstration die Bevölkerung der Stadt auf die Gefahr aufmerksam zu machen: Sie ziehen mit Schildern durch die Stadt und laden zu einer Diskussionsrunde ins Amphitheater ein. Doch zur grossen Enttäuschung der Kinder kommt kein Erwachsener ans vereinbarte Treffen. Die grauen Herren hingegen sind nun hellwach geworden. Der Entscheid fällt: Momo ist gefährlich und muss beseitigt werden. Das erfährt zufällig Beppo, der insgeheim einer nächtlichen Verhandlung auf einer Kehrichtablage beiwohnt. Dort wird zunächst ein Gerichtsverfahren gegen den Agenten BLW/553/c durchgeführt, dessen Ergebnis das Todesurteil wegen Hochverrats ist. Die Hinrichtung geschieht dadurch, dass dem Agenten die Zigarre und die Aktentasche weggenommen werden: Augenblicklich löst er sich in nichts auf. Das Leben der grauen Herren muss also, so der Schluss Beppos, in den Zigarren liegen, und in den Aktentaschen befindet sich vielleicht ein Vorrat an Zigarren. Doch lange kann Beppo nicht darüber nachdenken, denn die grauen Herren fahren mit ihren grauen Wagen los, um Momo im Amphitheater gefangen zu nehmen. Beppo schwingt sich verzweifelt auf sein Fahrrad, um sie zu warnen. Aber als er ankommt, ist sie bereits verschwunden.

Er weiss jedoch nicht, dass sie nicht gefangen wurde. Sie wurde vorher schon abgeholt, und zwar von einer Schildkröte namens Kassiopeia. Ein schönes Gleichnis:Wer könnte besser gegen Eile und Geschäftigkeit kämpfen als eine Schildkröte! Und mit Kassiopeia werden sich die Zeitverhältnisse mehrmals überraschend umkehren. Kassiopeia spricht nicht. Sie antwortet jedoch, indem auf ihrem Rückenpanzer leuchtende Buchstaben in Kurzsätzen erscheinen; und so kann Momo mit ihr kommunizieren. Kassiopeia wurde 
beauftragt (unklar ist, von wem), Momo in Sicherheit zu bringen. Sie ziehen nun im Schildkrötentempo durch die Stadt, während die grauen Herren in ihren Wagen hastig durch die Stadt rasen, auf der Suche nach Momo. Nie jedoch finden sie das Mädchen, denn in aller Langsamkeit entgeht die Schildkröte den rasenden Wagen, weil sie eine halbe Stunde im Voraus weiss, was passieren wird. So kommen Kassiopeia und Momo langsam, aber sicher (das gilt hier im wörtlichen Sinne!) in einen Bereich der Stadt, in dem alles seltsam wird, einen Bereich am Rande der Zeit und sogar ausserhalb der Zeit. Die rasenden Wagen kommen plötzlich nicht mehr vom Fleck, während sie selbst unglaublich schnell vorankommen. "Je langsamer, desto schneller», heisst es auf dem Rückenpanzer. ${ }^{2}$ Der Weg geht nun durch die Niemals-Gasse: Hier kommt man nur noch voran, indem man rückwärts geht, weil die Zeit dort auch rückwärts geht. Und diese Gasse führt zum Nirgend-Haus: Durch eine kleine Tür mit der Aufschrift "Meister Secundus Minutius Hora» treten sie in das Haus hinein; jetzt sind sie vor den grauen Herren in Sicherheit.

\section{Das Geheimnis Meister Horas}

Da die Verfolgung gescheitert ist, hält der Vorstand der grauen Herren eine Sitzung ab, um zu beraten, was nun zu machen sei. Sie sitzen um einen Konferenztisch. "Die Stimmung - soweit man bei diesen Herren überhaupt von so etwas wie Stimmung reden konnte - war allgemein gedrückt«. (149) Was schon lange befürchtet wurde, habe sich ereignet: Anscheinend habe "der Betreffende« Momo geholfen. Sonst hätte sie nicht entkommen können. Er habe sie in Sicherheit gebracht, in den Bereich ausserhalb der Zeit. Sie werde jedoch zurückkommen, und dann müssten sie angemessen handeln. Beschlossen wird schliesslich, Momo all ihre Freunde wegzunehmen. So, in derVereinsamung, werde man sie schon zur Mitarbeit zwingen können. Da sie jetzt ja den Weg zum »Betreffenden« wisse, werde sie die grauen Herren zu ihm führen und ihnen damit helfen, endgültig in den totalen Besitz der Zeit zu kommen.

Der "Betreffende«, von dem die grauen Herren nur mit grosser Ehrfurcht und Zurückhaltung sprechen, ist Meister Hora, mit dem Momo in einem grossen Saal, der voll von unendlich vielen, unterschiedlichen Uhren ist, Bekanntschaft gemacht hat. Es ist eine seltsame, väterliche oder grossväterliche Figur, manchmal ganz alt und manchmal wieder ganz jung aussehend! Von ihm vernimmt Momo

2 S. 259. Ein Motto, das der Universität ab und zu auch gut täte! 
nun viel: dass die grauen Herren sie verfolgen, weil sie den einen Agenten zum Verrat gebracht habe und die Wahrheit aufdecken wolle; dass die grauen Zigarren getrocknete und zerriebene, also eigentlich tote Zeit enthalten und die grauen Herren beim Rauchen tote Zeit einnehmen; dass sie deshalb auch immer Vorrat an Zigarren brauchen, den sie in ihren Aktentaschen tragen; dass sie aber auch stets in der Zeit-Spar-Kasse neuen Zigarrenvorrat holen müssen. Aber wichtiger ist, was Momo über die Zeit erfährt. Nachdem sie ein schwieriges Rätsel gelöst hat, in dem es um Vergangenheit, Gegenwart und Zukunft geht, darf ihr ein Geheimnis anvertraut werden: »Hier aus dem Nirgend-Haus in der Niemals-Gasse kommt die Zeit aller Menschen«. (176) Auf die Frage, ob denn Meister Hora die Zeit selbst mache, antwortet er: "Nein, mein Kind, ich bin nur der Verwalter. Meine Pflicht ist es, jedem Menschen die Zeit zuzuteilen, die ihm bestimmt ist«. (ebd.) Auf die Frage, ob er es denn nicht einfach einrichten könne, dass die Zeit-Diebe den Menschen keine Zeit mehr stehlen können, folgt die Antwort, das könne er nicht, weil die Menschen selbst dafür zuständig seien, wie sie mit Zeit umgehen: „Denn so, wie ihr Augen habt, um das Licht zu sehen, und Ohren, um Klänge zu hören, so habt ihr ein Herz, um damit die Zeit wahrzunehmen.... Aber es gibt leider blinde und taube Herzen, die nichts wahrnehmen, obwohl sie schlagen«. (ebd.)

\section{Das Geheimnis der Stunden-Blumen}

Plötzlich fragt Meister Hora: »Möchtest du sehen, woher die Zeit kommt?« (178) Momo flüstert "Ja«. Meister Hora nimmt sie in die Arme und geht einen langen Weg, bis sie unter einer gewaltigen runden Kuppel mit goldener Dämmerung stehen. Aus einer runden Öffnung in der Mitte der Kuppel fällt eine Lichtsäule senkrecht auf einen ebenfalls kreisrunden, dunklen Wasserteich hinunter. In der Lichtsäule bewegt sich langsam ein ungeheures Pendel über dem schwarzen Wasserspiegel hin und her. Mit jeder Bewegung des Pendels taucht eine grosse Blütenknospe aus dem Wasser. »Je näher das Pendel kam, desto weiter öffnete sie sich, bis sie schliesslich voll erblüht auf dem Wasserspiegel lag. Es war eine Blüte von solcher Herrlichkeit, wie Momo noch nie zuvor eine gesehen hatte«. (179) Während sich das Pendel langsam wieder entfernt, beobachtet Momo mit Bestürzung, dass die herrliche Blume langsam verwelkt. "Als das Pendel über der Mitte des schwarzen Teiches angekommen war, hatte die herrliche Blüte sich vollkommen aufgelöst. Gleichzeitig aber 
begann auf der gegenliegenden Seite eine Knospe aus dem dunklen Wasser aufzusteigen". (179f.) Daraus wird eine noch herrlichere Blüte, die dann bei der nächsten Bewegung des Pendels auch wieder verwelkt, während anderswo eine nochmals ganz andere, noch schönere Blume aufgeht, um auch wieder zu verwelken, usw. Zugleich vernimmt Momo nun auch einen Klang, Musik, Harmonien, und dann nach und nach Worte, "Worte einer Sprache, die sie noch nie vernommen hatte und die sie doch verstand " (182), Worte, die an sie gerichtet waren. Licht, Musik, Worte: all das zusammen erfährt Momo als höchste Beglückung, und zwar nicht irgendwo: »... wo war ich denn? «, fragt Momo. »In deinem eigenen Herzen«, antwortet Meister Hora (183).

\section{Das Geheimnis der Angst, die in Mut umschlägt}

Momo wacht im Amphitheater auf. Aber sie bleibt weiterhin beglückt, voll von der Farbenpracht der Stunden-Blumen und von der sie begleitenden Musik. Sie will ihre Freunde daran teilhaben lassen und macht sich deshalb auf die Suche. Doch da muss sie Enttäuschung auf Enttäuschung erleben. Nach langem Suchen gelingt es ihr, auf die Spur von Gigi zu kommen. Doch er hat keine Zeit mehr für sie: er ist ein beliebter Erzähler geworden, der für seine Auftritte von Ort zu Ort hascht. Beppo ist nicht auffindbar; der Leser vernimmt, dass ihm ein grauer Herr versprochen hat, dass Momo befreit werde, wenn er beim Strassenkehren hunderttausend Stunden einsparen könne, und so kehrt er nun pausenlos alle Strassen in grösster Eile, wie besessen. Auch die Kinder sind unerreichbar: Sie sind in Kinder-Depots untergebracht und haben "Gesichter wie kleine Zeit-Sparer« (207). Momo sucht andere Freunde auf, aber alle sind Opfer der grauen Herren geworden. Beim Umherirren verliert sie auch noch Kassiopeia. Momo vereinsamt und verzweifelt, wird hilflos und bekommt Angst, möchte nur noch fliehen.

In tiefstem Leiden fühlt sie jedoch plötzlich eine seltsame Veränderung: "Das Gefühl der Angst und Hilflosigkeit war so gross geworden, dass es plötzlich umschlug und sich ins Gegenteil verwandelte. Es war durchgestanden. Sie fühlte sich nun so mutig und zuversichtlich, als ob keine Macht der Welt ihr etwas anhaben könnte ...«. (246) Anstatt zu fliehen, will sie nun den grauen Herren standhalten. Die Auseinandersetzung geschieht denn auch gleich: Auf einem verlorenen, leeren Platz wird sie in der Nacht umzingelt von den grauen Wagen. Sie steht im Licht der Scheinwerfer um 
sie herum und wird von Stimmen im Dunkeln verhört, bedroht, ausgefragt, während sich eine grosse Kälte ausbreitet, an der sie fast erfriert. Als die grauen Herren erfahren, dass nicht sie den Weg zu Meister Hora weiss, sondern Kassiopeia, stürzen sie weg, machen sich auf die Suche nach der Schildkröte. Doch diese taucht plötzlich bei der einsamen Momo auf. Sie entscheiden, zu Meister Hora zu gehen, machen sich auf den Weg, merken aber nicht, dass sie von einigen grauen Herren gefolgt werden, die lauernd in der Nähe geblieben waren. Es werden nun immer mehr, die ihnen auf der Schliche sind, und dauernd stossen weitere hinzu. Als Momo zur Niemals-Gasse kommt und sich umdreht, sieht sie mit Schrecken die grosse Horde von grauen Herren. Diese können zwar nicht ins Nirgend-Haus eintreten, weil sie die rückwärtsgehende Zeit in der Niemals-Gasse davon abhält. Sie umzingeln nun aber das Haus in dichten Reihen und mit dem Qualm ihrer Zigarren verbreitet sich immer mehr tote Zeit um das Haus herum. Meister Hora ist zutiefst besorgt, denn diese tote Zeit wird die den Menschen notwendige Zeit, die vom Nirgend-Haus ausgeht, vergiften. Es muss unbedingt etwas geschehen.

\section{Das Geheimnis des Sieges}

Nach längerer Beratung schlägt Meister Hora Momo einen Auftrag vor. Er wird für eine Stunde die Zeit stillstehen lassen, was dazu führen wird, dass er selbst einschlafen wird. Momo wird eine StundenBlume bekommen und handeln müssen, bevor diese verwelkt. Sie hat also eine Stunde Zeit. Wenn die grauen Herren merken werden, dass die Zeit still steht, werden sie zu ihrem Vorrat in der Zeit-SparKasse rennen. Momo muss ihnen folgen, dafür sorgen, dass sie nicht zu ihrem Vorrat kommen und, sobald sie sich alle in nichts aufgelöst haben, den Vorrat öffnen. Dieser besteht nämlich aus eingefrorenen Stunden-Blumen. Momo soll sie befreien, damit sie wieder den Menschen zuteil werden.

Momo nimmt den Auftrag an, die Stunden-Blume in der Hand und Kassiopeia unter dem Arm (denn man wird schnell laufen müssen!). Plötzlich steht die Zeit still und nichts mehr bewegt sich, alles versteinert um Momo herum ... ausser der Horde der grauen Herren, die plötzlich das Nirgend-Haus einnehmen, denn nichts schützt es mehr vor ihnen. Als sie aber merken, dass die Zeit still steht, wenden sie sich ab. Das Rennen zur Zeit-Spar-Kasse wird zu einem unglaublichen Gemetzel: Je schneller sie rennen, desto mehr 
Zeit und Zigarren verbrauchen sie. Beim Rennen reissen sie einander die Zigarren aus dem Mund, stehlen einander die Aktentaschen, kämpfen verbissen um jede Zigarre, bis der eine oder der andere sich in nichts auflöst. Momo gelingt es mühsam, ihnen zu folgen. Als sie beim Vorrat ankommen, sind es nur noch relativ wenige. Es geht hinunter in ein unterirdisches Gebäude. Die restlichen grauen Herren setzen sich an einen Tisch, um zu überlegen, wie man am sparsamsten mit dem Vorrat umgehen soll, denn niemand weiss, wie lange die Zeit still stehen wird. Die Stunden-Blume in Momos Hand beginnt bereits zu welken. Die Tür zur Vorratskammer steht offen, und weil alles still steht, lässt sie sich auch nicht schliessen. Doch Kassiopeias Panzer leuchtet auf:»Mit der Blume berühren!» (290) Momo kriecht bis zur Tür und schliesst sie mit der Stunden-Blume. Die grauen Herren ahnen schnell ihr Unheil, versuchen nun, dem Mädchen die Blume zu entreissen. Mit Hilfe Kassiopeias entkommt ihnen Momo immer. Die grauen Herren kämpfen untereinander, weil jeder die rettende Blume für sich haben will, und so lösen sich schliesslich die letzten in nichts auf. Der Feind ist besiegt. Mit der schon fast ganz verwelkten Blume öffnet Momo wieder dieVorratskammer: Es wird wärmer, wie in einem Treibhaus, und eine Art Frühlingswind beginnt zu wehen. "Wolken von Stunden-Blumen wirbelten um sie her und an ihr vorüber«. (293) Lauter befreite Zeit, die wie ein starker Strom Momo aufhebt und davonträgt, bis zum Amphitheater.

\section{Ein paar Schlussgedanken}

Mit einer einfachen Stunden-Blume hat Momo die grauen Herren besiegt, die Zeit aus dem eiskalten Gefängnis der Zeit-Spar-Kasse befreit. Weil die Zeit nun nicht mehr gespart wird, ist sie plötzlich wieder in Fülle da. "Überall standen Leute, plauderten freundlich miteinander und erkundigten sich ausführlich nach dem gegenseitigen Wohlergehen. ... die Ärzte hatten jetzt Zeit, sich jedem ihrer Patienten ausführlich zu widmen. Die Arbeiter konnten ruhig und mit Liebe zur Sache arbeiten, denn es kam nicht mehr darauf an, möglichst viel in möglichst kurzer Zeit fertig zu bringen«. (296)

Diese Wandlung lässt sich in Beppos Arbeitsauffassung beim Strassenkehren konkretisieren, die wie ein Gegenmodell zu aller (auch universitären?) Planungssucht ist: "Manchmal hat man eine sehr lange Strasse vor sich. Man denkt, die ist so schrecklich lang; das kann man niemals schaffen, denkt man. ... Und dann fängt man an sich zu eilen. Und man eilt sich immer mehr. Jedes Mal, wenn man 
aufblickt, sieht man, dass es gar nicht weniger wird, was noch vor einem liegt. ... man kriegt es mit der Angst, und zum Schluss ist man ganz ausser Puste und kann nicht mehr. Und die Strasse liegt immer noch vor einem. So darf man es nicht machen«. (38)

Wie ist es denn in Angriff zu nehmen? Beppos Weisheit lautet: "Man darf nie an die ganze Strasse auf einmal denken, verstehst du? Man muss nur an den nächsten Schritt denken, an den nächsten Atemzug, an den nächsten Besenstrich. Und immer wieder an den nächsten.... Dann macht es Freude; das ist wichtig, dann macht man seine Sache gut. Und so soll es sein. ... Auf einmal merkt man, dass man Schritt für Schritt die ganze Strasse gemacht hat. Man hat gar nicht gemerkt wie ...«. (38f.)

Das würde aber auch heissen, dass es bei einem solchen Zeitverständnis Leute geben könnte, die verschwenderisch Zeit geben. Man hat dieses Geben zwar kaum im Griff. Es geschieht unvorhergesehen, es widerfährt einem plötzlich, wie ein kleines Wunder. "Pequeños milagros", nach der Überschrift eines schönen lateinamerikanischen Films. Die Planungsherren jedoch wollen solches kaum wahrhaben: Menschen haben effizient zu arbeiten, Zeit sparend; und laufende Evaluationen und rollende Planungen erweisen, dass noch mehr drin liegt, dass man es zu noch mehr in noch weniger Zeit bringen kann.

So haben es solche Menschen schwer, die nicht ins Schema der Planer passen. Und doch gibt es sie. Es widerfährt ihnen unversehens, dass sie Zeit geben, manchmal sogar an der Universität, wo man doch Wichtigeres treiben sollte, wo man dafür sorgen sollte, es zu etwas zu bringen (denkt doch an die vielen Rankings!). Und wenn die Planer den Menschen die Zeit stehlen, dann können diese queren Menschen manchmal sogar diese Zeit zurückgeben. Es ist kein Ding der Unmöglichkeit, wohl aber vielleicht die Frucht einer besonderen Gnade. Man wird ja nicht sagen können, dass solche Leute sich die Zeit nehmen, Zeit zu geben, denn man wüsste gar nicht, woher sie sie nähmen. Sie haben keinen zusätzlichen Zeitvorrat! Anscheinend haben sie Zeit zum Verschenken geschenkt bekommen. Vielleicht durften sie manchmal Momos Zuhören erleben, und vor diesem haben ja die grauen Herren bekanntlich keinen Bestand .... 


\section{Pierre Bühler}

Am Anfang des Buches steht als Motto ein Zitat "nach einem alten irischen Kinderlied«:

Im Dunkel scheint dein Licht.

Woher, ich weiss es nicht.

Es scheint so nah und doch so fern.

Ich weiss nicht, wie du heisst.

Was du auch immer seist:

Schimmere, schimmere, kleiner Stern!

— Dr. Pierre Bühler ist Professor für Systematische Theologie, insbesondere Hermeneutik und Fundamentaltheologie an der Universität Zürich. 